\title{
Lentigo Maligna of the Face: The Importance of Clinical, Dermoscopic, and Histological Correlation
}

\author{
Giovana Serrão Fensterseifer ${ }^{1}$, Ana Paula Lodi ${ }^{1}$, Manuela Lima Dantas ${ }^{1}$, \\ Ana Letícia Boff ${ }^{2}$, Louise Lovatto ${ }^{3}$
}

1 Dermatology, Santa Casa de Porto Alegre, Brazil

2 Dermatopathology, Santa Casa de Porto Alegre, Brazil

3 Pigmented Lesions Clinic, Santa Casa de Porto Alegre, Brazil

Key words: lentigo maligna, pigmented lesions of the face, dermoscopy, histopathology

Citation: Serrão Fensterseifer G, Lodi AP, Lima Dantas M, Boff AL, Lovatto L. Lentigo maligna of the face: the importance of clinical, dermoscopic, and histological correlation. Dermatol Pract Concept. 2019;9(4):292-294. DOI: https://doi.org/10.5826/dpc.0904a08

Accepted: July 21, 2019; Published: October 31, 2019

Copyright: (02019 Serrão Fensterseifer et al. This is an open-access article distributed under the terms of the Creative Commons Attribution License, which permits unrestricted use, distribution, and reproduction in any medium, provided the original author and source are credited.

Funding: None.

Competing interests: The authors have no conflicts of interest to disclose.

Authorship: All authors have contributed significantly to this publication.

Corresponding author: Dr. Giovana Serrão Fensterseifer, Rua Professor Annes Dias, 135-Hospital Santa Clara, Santa Casa Porto Alegre, Brazil. Email: gfensterseifer@gmail.com

\section{Introduction}

Lentigo maligna (LM) is a type of in situ melanoma that usually arises in elderly people with sun-damaged skin. The accurate diagnosis of these lesions is difficult because, besides having other pigmented macules in the differential diagnosis, the histopathology has subtle features that can be confused with junctional melanocytic nevi (JMN).

\section{Case Presentation}

We present 4 cases of patients who had LM on the face, initially being misdiagnosed as JMN by biopsy of the lesions. Patient 1, a 59-year-old man, presented with a brown lesion on the face at the same spot where he had a lesion removed previously (Figure 1, A and C). Asymmetric pigmentation with gray dots around the follicle was seen on dermoscopy (Figure 1B).

Patient 2, aged 74 years, presented with new lesion on the face, revealing asymmetric gray pigment around the follicle and rhomboidal structures on dermoscopy (Figure 2).
Patient 3, aged 58 years, presented with a lesion on the face that had been present for 3 years. Multiple gray dots, some of them distributed around the follicle, were seen on dermoscopy (Figure 3).

Patient 4, aged 60 years, presented with a lesion on the forehead that had been gradually growing. It presented asymmetric gray pigmentation around the follicle, rhomboidal structures, and circle-within-circle structures on dermoscopy (Figure 4).

\section{Conclusions}

When evaluating pigmented macules on the face, one should keep in mind the differential diagnosis, such as solar lentigo, seborrheic keratosis, pigmented actinic keratosis (PAK), and lichen planus-like keratosis (LPLK). Dermoscopy can give some clues to help with the accurate diagnosis. Stolz et al described a model of the dermoscopy progression for LM: it initially has asymmetric pigmentation and dots around the follicle, evolving to rhomboidal structures and then forming homogeneous pigmented areas and obliteration of the follic- 


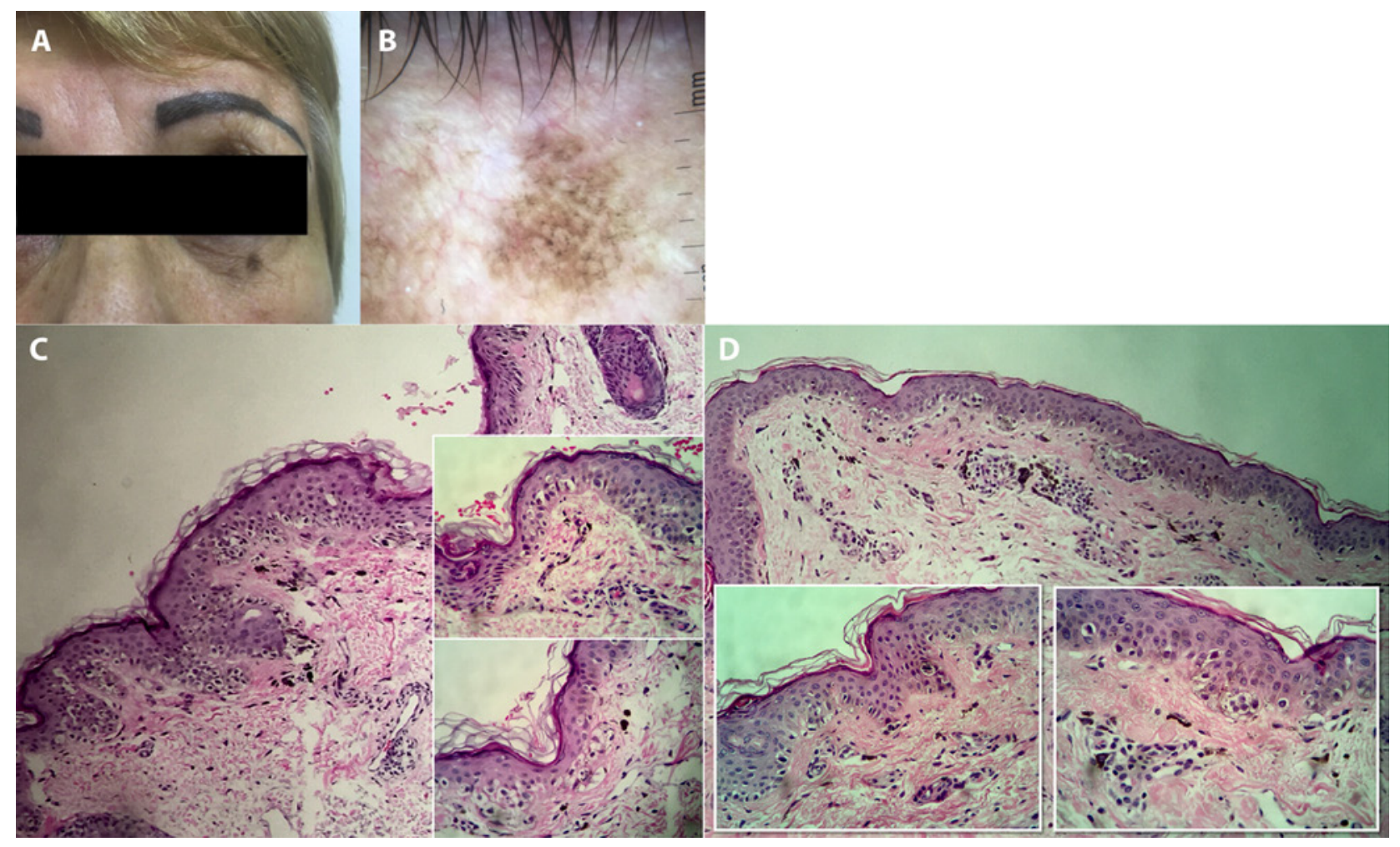

Figure 1. Patient 1. (A) Clinical image. (B) Dermoscopy. (C) The first excisional biopsy was initially misdiagnosed as junctional melanocytic nevi. Histopathology ( $\times 40$ and $\times 400)$ shows lentiginous proliferation of atypical melanocytes (pleomorphic and hyperchromic nucleus) involving acrosyringia and some pagetoid proliferation. Some melanocytes are arranged in nests. There are melanophages in the papillary dermis. (D) Biopsy of the recurrent lesion. Histopathology $(\times 40$ and $\times 400)$ shows even more subtle findings than the first one: lentiginous proliferation of melanocytes with bigger, hyperchromic, and irregular-shaped nucleus; melanocytes arranged in nests; and melanophages in the papillary dermis. [Copyright: @2019 Serrão Fensterseifer et al.]

ular openings [1]. The existence of gray pigmentation (that can be arranged in an annular-granular pattern) is also an important criterion for the suspicion of LM. Nevertheless, many LM dermoscopy criteria can also be seen in other lesions of the face, especially in PAK and LPLK. The histopathology of LM is characterized, initially, by subtle proliferation of slightly larger-dimensioned melanocytes at the basal layer of the epidermis of photo-damaged skin. These melanocytes are not equidistant from each other; they can be agglomerated and involve adnexa. At the next stage, there are more features of a malignant melanocytic lesion: pagetoid proliferation, melanocytes with hyperpigmented and angulated nucleus aligned in the basal layer, and involvement of the deeper portion of the follicles. At the third stage, the melanocytes can be arranged in nests, resembling a junctional nevus. At this stage, it is important to pay attention to the involvement

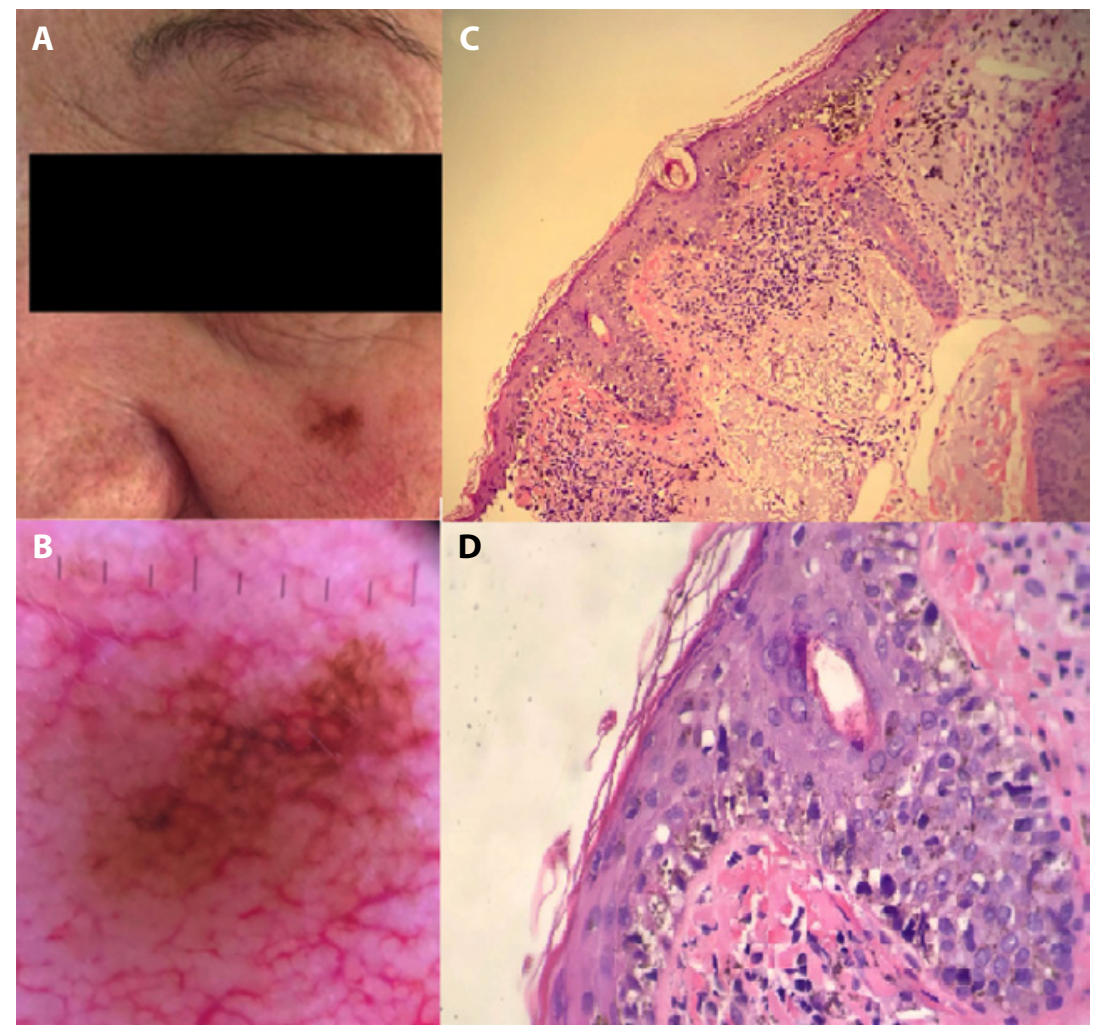

Figure 2. Patient 2. (A) Clinical image. (B) Dermoscopy. (C) Histopathology examination $(\times 40)$ shows continuous lentiginous proliferation and some melanocytes arranged in junctional nests. (D) Higher magnification $(\times 400)$ shows atypical and a few pagetoid melanocytes. [Copyright: @2019 Serrão Fensterseifer et al.] 
of adnexa, to the discrete pagetoid proliferation, to the disposal of the nests parallel to the epidermis, and to the shape of the melanocytes [2]. Moreover, it is well known that nevi on sun-exposed skin of elderly people are usually intradermal nevi, being pink, domeshaped papules.

In conclusion, the differential diagnosis of recent pigmented macules on sun-exposed skin of elderly individuals is usually between LM and nonmelanocytic lesions; it is always prudent to reevaluate pathology reports of JMN, followed by an excisional biopsy of these lesions.

\section{References}

1. Stolz W, Schiffner R, Burgdorf WH. Dermatoscopy for facial pigmented skin lesions. Clin Dermatol. 2002;20(3):276278.

2. Massi G, LeBoit PE. Lentigo maligna. In: Massi G, LeBoit PE. Histological Diagnosis of Nevi and Melanoma. 2nd ed. London: Springer; 2014:437-444.
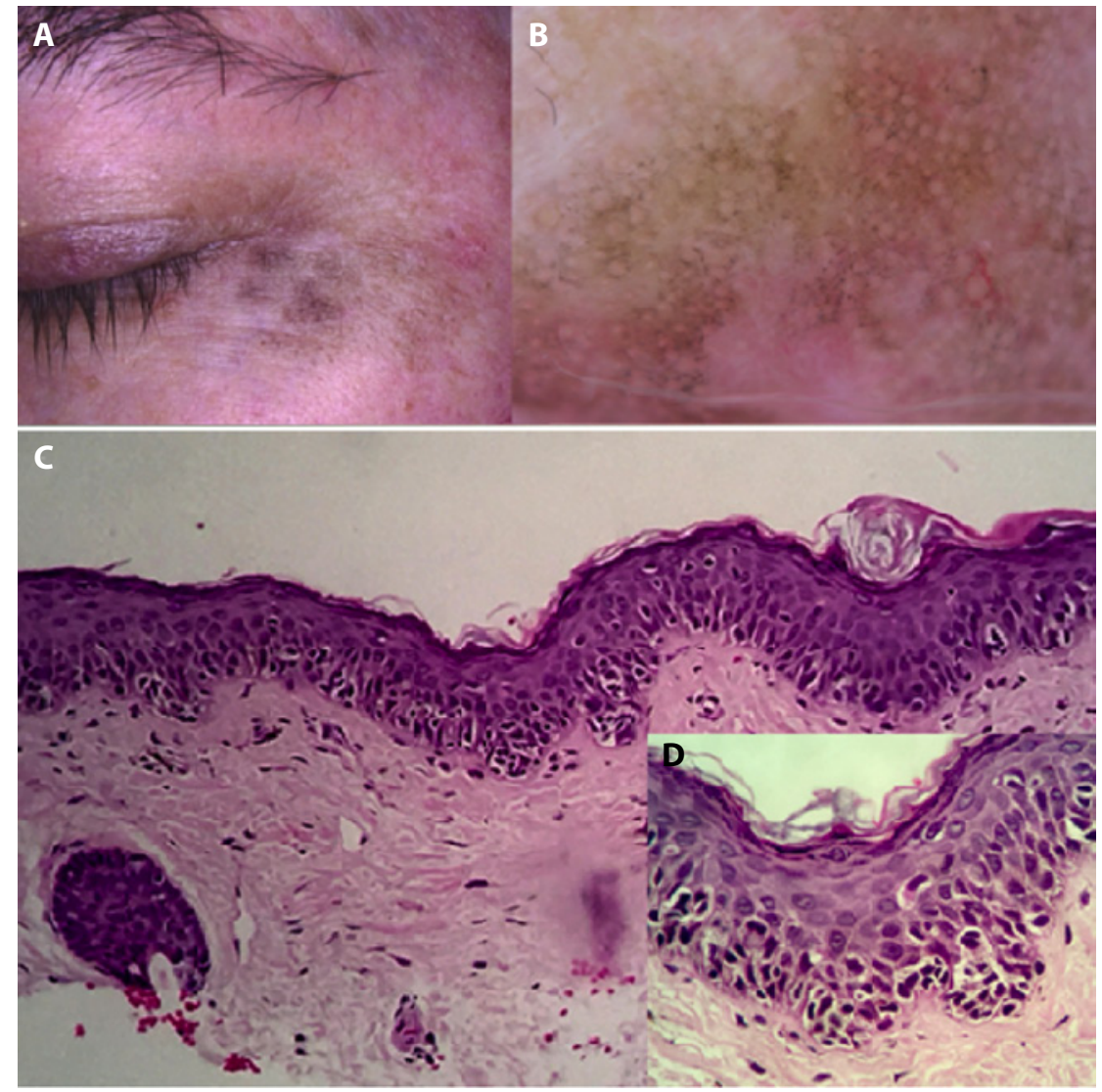

Figure 3. Patient 3. (A) Clinical image. (B) Dermoscopy. (C) Histopathology examination $(\times 40)$ shows continuous lentiginous proliferation and some pagetoid melanocytes. (D) Insert shows atypical melanocytes arranged in nests. [Copyright: (02019 Serrão Fensterseifer et al.]
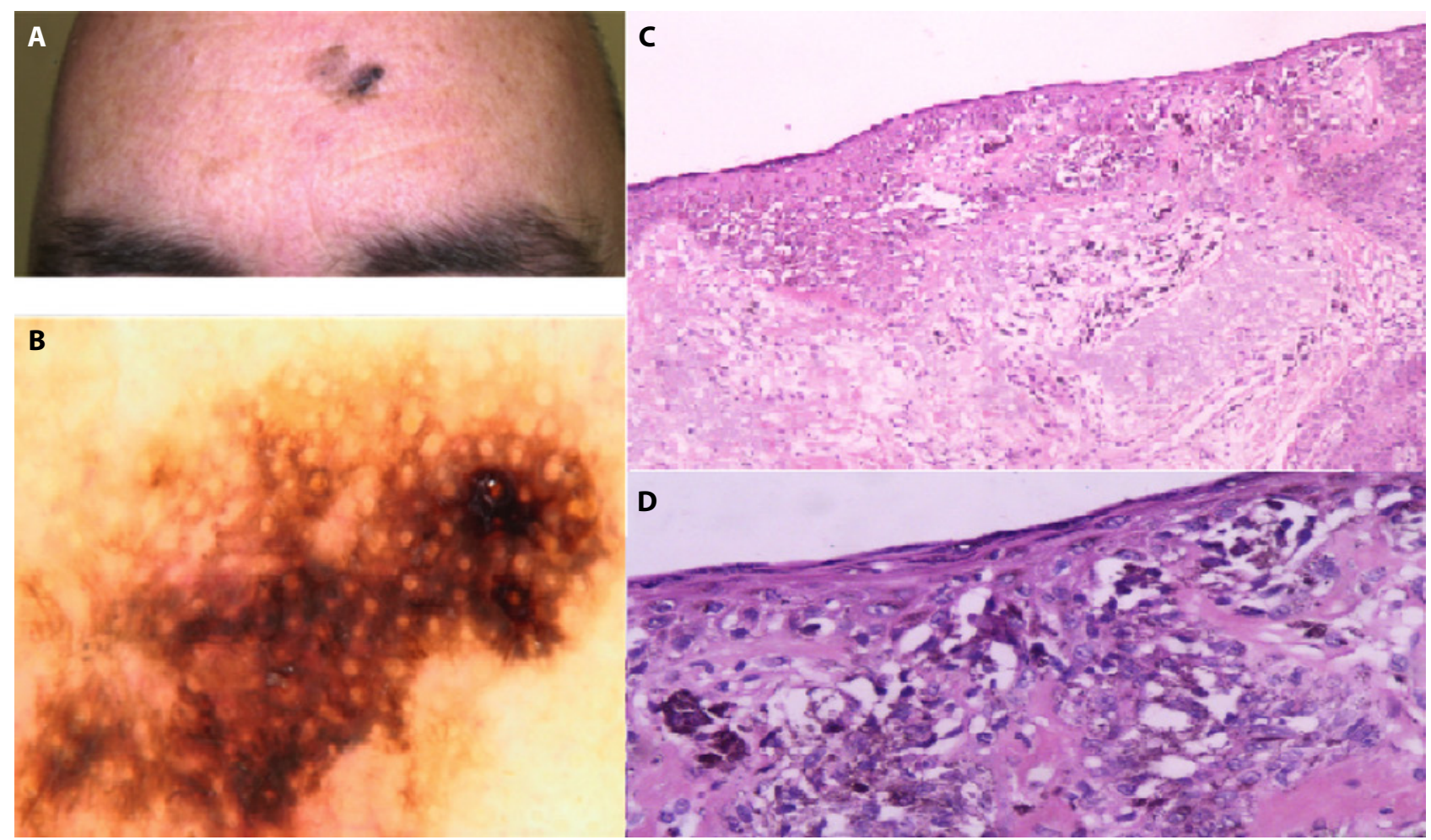

Figure 4. Patient 4. (A) Clinical image. (B) Dermoscopy. (C) Histopathology shows lentiginous proliferation and junctional nests. (D) Higher magnification $(\times 400)$ shows atypical melanocytes arranged in nests. [Copyright: @2019 Serrão Fensterseifer et al.] 